\title{
Analysis of Motion Error of Planar Linkage Mechanism with Three-bar Composite Hinge with Clearance
}

\author{
Chong Liu ${ }^{a}$, Xinyuan Zhang ${ }^{b}$ and Yunpeng Xia ${ }^{c}$ \\ School of Mechanical and Electrical Engineering, Harbin Engineering University, China. \\ a Corresponding author: liuchong@hrbeu.edu.cn \\ bzhangxinyuanbest@163.com \\ c heuxiayunpeng@163.com
}

Keywords: Composite hinge zero displacement method; continuous contact model; matrix method; motion accuracy.

\begin{abstract}
Considering the problem of the movement accuracy of the three-bar composite planar linkage mechanism considering the hinge clearance, this paper applies the "hybrid zero displacement method" combined with the continuous contact model and the matrix method to analyze the motion error of the composite hinge plane linkage mechanism, finally using a certain project as an example of the six-bar locking mechanism in the middle plane, the effects of the gap between the mechanism and the rod length on the movement parameters of the locking mechanism are analyzed. The analysis results show that the motion error model based on the composite hinge zero offset method has a good analysis result, and it has a certain guiding significance for practical engineering application and theoretical research.
\end{abstract}

\section{Introduction}

Because of its high safety and reliability, the connecting rod mechanism is widely used in the locking system. At the same time, its movement accuracy requirements are also getting higher and higher. Therefore, more and more scholars have begun to pay attention to the problem of the motion accuracy of the multi-linkage mechanism. In the analysis of the motion accuracy of the multi-bar mechanism, most scholars use the continuous contact model for the gap in the mechanism's motion pair. The continuous contact model considers the auxiliary elements of the movement to keep in continuous contact and regards the clearance vector as a massless rod [1-3]. For the compound hinge problem existing in the multi-linkage mechanism, the mechanism topology can't use some of the theorems in graph theory due to the presence of polygons. For this reason, some new algorithms have been developed to solve this problem, for example, the two-color topography method in which the components and the motion pairs of the mechanism are expressed as the vertices of the undirected graph, or the components are considered as vertices and the topological structure of the mechanism is abstracted into a connected undirected graph method with the connecting points as edges, but the two methods make the organization's expression more complicated, which is not conducive to computer programming analysis. Therefore, in view of this deficiency, this paper uses the "hybrid zero displacement method [4]" to analyze the problem of the composite hinge in the multi-linkage mechanism and construct a new kinematic chain and topology diagram of the mechanism. On this basis, the continuous contact model was used to establish the effective length model of the composite hinge with clearance, and to further refine the mechanism motion error analysis model.

\section{Topological Description of Composite Hinge Mechanism with Composite Hinge}

The kinematic chain is a constraint system composed of components and motion pairs. Topological graphs represent the components of a kinematic chain with vertices and represent the mechanism of motion pairs with line segments. The six-bar linkage's kinematic chain and topology are depicted in Figure 1. 


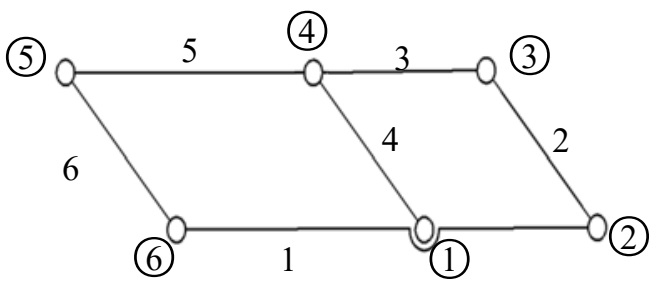

(a) Kinematic chain

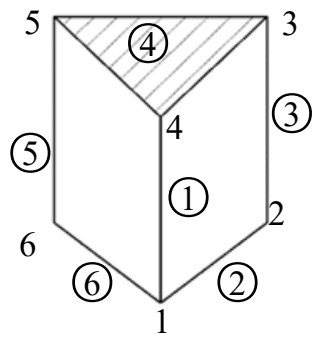

(b) Topology diagram

Figure 1. Kinematic chain and topology of six-bar linkage

The concept of nodes is first introduced here, and two or more multiple joints (movement pairs or components) are called nodes. In the kinematic chain shown in Figure 1a in this section, node (4) is a multiple joint with 2 rotary pairs. The multiple joint zero offset method is used to shift the movement auxiliary elements of the rod 3 in position of the node (4), and then connected with the movement auxiliary elements of positions 4 and 5 in the position of the node (4) respectively, so as to obtain two single hinges as shown in Fig. 2. The pair of movements, where the distance between the two rotation pairs is zero, the topological diagram of the new kinematic chain is shown in Figure $2 \mathrm{~b}$. Comparing Fig. $1 \mathrm{~b}$ and Fig. 2b, it can be found that the polygon representing the multiple joint disassembles one edge, thereby facilitating subsequent establishment of the joint clearance model. Here, the splitting of the composite hinge is based on the definition of the rod group, the rod group splitting theorem [5] and the actual operating conditions, which will not be elaborated here.

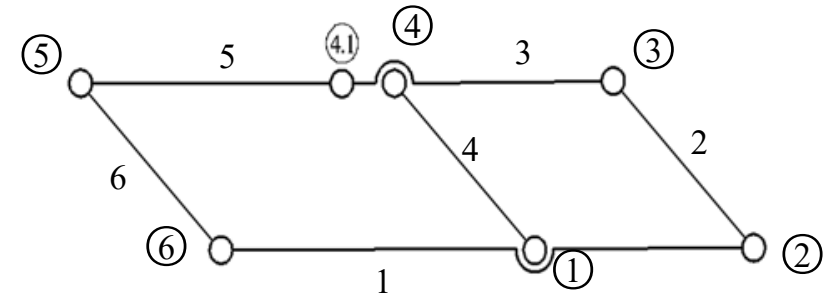

(a) Kinematic chain

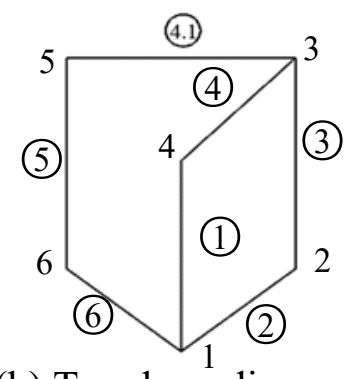

(b) Topology diagram

Figure 2. Kinematic chain and topology of six-bar linkage

\section{Mechanism Motion Error Analysis Model}

\subsection{Composite Hinge Model with Clearance Model}

Based on the composite hinge model and the continuous contact model [3], the effective length model with gap in the mechanism can be described as shown in Figure 3, where $r_{i}, \theta_{i}, \alpha_{i}, l_{i}$ represents the gap radius, the rod movement angle, and the gap position angle. Due to the limited space, only the rod length models of rods 3, 4 and 5 and the corresponding gap angles are expressed as shown in equations (1) and (2).

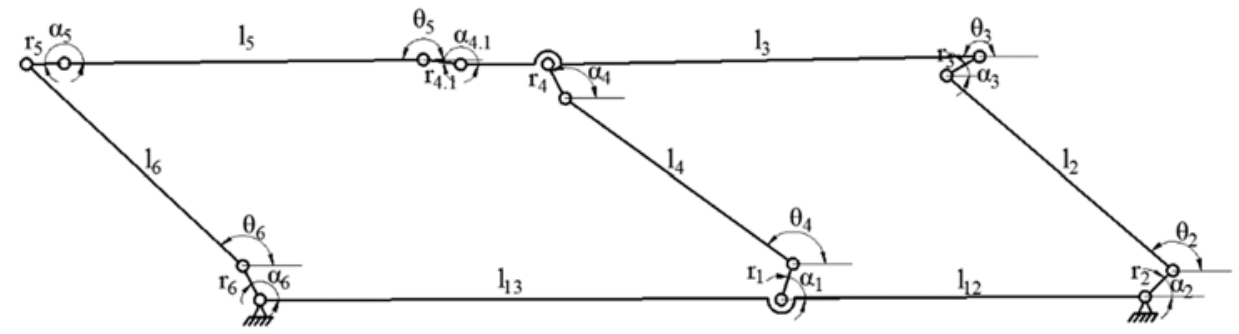

Figure 3. Model of a six-bar mechanism with a composite hinge gap 


$$
\begin{aligned}
& l_{3}=l_{3}^{\prime}-r_{3} \cos \left(\theta_{3}-\alpha_{3}\right)+r_{4.1} \cos \left(\theta_{5}+\alpha_{4.1}\right) \\
& l_{4}=l_{4}^{\prime}-r_{1} \cos \left(\theta_{12}-\alpha_{1}\right)+r_{4} \cos \left(\theta_{5}+\alpha_{4}\right) \\
& l_{5}=l_{5}^{\prime}+r_{4.1} \cos \left(\theta_{5}+\alpha_{4.1}\right)+r_{5} \cos \left(\theta_{6}+\alpha_{5}\right)
\end{aligned}
$$

Where $r_{i}$ is determined by the fit clearance, and the clearance angle is obtained by solving the equation of motion. It is obtained by the following formula.

$$
\begin{aligned}
& \alpha_{4}=\arccos \frac{\sqrt{4 l_{5}^{\prime 2}+r_{4}^{2}}-2 l_{5}^{\prime}}{2 r_{4}}-\theta_{5} \\
& \alpha_{4.1}=\arccos \frac{\sqrt{4 l_{5}^{\prime 2}+r_{4.1}^{2}}-2 l_{5}^{\prime}}{2 r_{4.1}}-\theta_{5}
\end{aligned}
$$

\subsection{Comprehensive Consideration of Rod Length Error and Hinge Clearance Model}

With the establishment of a model with a gap-length bar, the effective length of each bar can be calculated. Based on the effective length of the bar, a matrix analysis method is used to establish the analysis model of the movement accuracy of the integrated gap and bar length error factors. Let the output parameters of the linkage mechanism and the random variables affecting the output parameters satisfy the following equation [6]:

$$
F(Y, X, L)=0
$$

In the equation: $F=\left[f_{1}, f_{2}, f_{3}, \ldots, f_{\lambda}\right]^{T}$ is a vector composed of independent mechanism equations, $Y=\left[y_{1}, y_{1}, y_{1}, \ldots, y_{\lambda}\right]^{T}$ is a vector composed of mechanism output parameters, $X=\left[x_{1}, x_{2}, x_{3}, \ldots, x_{m}\right]^{T}$ is a vector composed of mechanism input parameters; $L=\left[l_{1}, l_{2}, l_{3}, \ldots, l_{\mathrm{n}}\right]^{T}$ is a vector composed of effective structure parameters of the mechanism.

From equation (5), the relationship between the output parameters of the mechanism, the input parameters, and the effective structure parameters can be obtained.

$$
Y=Y(X, L)
$$

Neglecting the elastic deformation of the member, the first-order Taylor of formula (4) is expanded at the nominal value, and further collated.

$$
\Delta Y=-\left[\frac{\delta F}{\delta Y}\right]^{-1}\left[\frac{\delta F}{\delta X} \Delta X+\frac{\delta F}{\delta L} \Delta L\right]
$$

The " $\Delta$ " in the equation represents the deviation, $\frac{\delta F}{\delta X}, \frac{\delta F}{\delta Y}, \frac{\delta F}{\delta L}$ are the Jacobian matrix.

formula (5) is used to differentiate time differentiation once and twice separately.

$$
\begin{gathered}
\Delta \dot{Y}=-A \Delta \dot{X}-A_{1} \Delta X-B_{1} \Delta L \\
\Delta \ddot{Y}=-A \Delta \ddot{X}-2 A_{1} \Delta \dot{X}-A_{2} \Delta X-B_{2} \Delta L
\end{gathered}
$$

Where 


$$
\begin{aligned}
& A=\left[\frac{\delta F}{\delta Y}\right]^{-1} \frac{\delta F}{\delta X}, B=\left[\frac{\delta F}{\delta Y}\right]^{-1} \frac{\delta F}{\delta L} ; \\
& A_{1}=\left[\frac{\delta F}{\delta Y}\right]^{-1}\left[\frac{d}{d t}\left(\frac{\delta F}{\delta X}\right)-\frac{d}{d t}\left(\frac{\delta F}{\delta Y}\right) A\right], B=\left[\frac{\delta F}{\delta Y}\right]^{-1}\left[\frac{d}{d t}\left(\frac{\delta F}{\delta L}\right)-\frac{d}{d t}\left(\frac{\delta F}{\delta Y}\right) B\right] ; \\
& A_{2}=\left[\frac{\delta F}{\delta Y}\right]^{-1}\left[\frac{d^{2}}{d t^{2}}\left(\frac{\delta F}{\delta X}\right)-\frac{d^{2}}{d t^{2}}\left(\frac{\delta F}{\delta Y}\right) A-2 \frac{d}{d t}\left(\frac{\delta F}{\delta Y}\right) A_{1}\right], B_{2}=\left[\frac{\delta F}{\delta Y}\right]^{-1}\left[\frac{d^{2}}{d t^{2}}\left(\frac{\delta F}{\delta L}\right)-\frac{d^{2}}{d t^{2}}\left(\frac{\delta F}{\delta Y}\right) B-2 \frac{d}{d t}\left(\frac{\delta F}{\delta Y}\right) B_{1}\right]
\end{aligned}
$$

Therefore, the mean of the error of the output parameters (displacement, velocity and acceleration) of the mechanism is respectively:

$$
\begin{gathered}
E(\Delta Y)=-A E(\Delta X)-B E(\Delta L) \\
E(\Delta \dot{Y})=-A E(\Delta \dot{X})-A_{1} E(\Delta X)-B_{1} E(\Delta L) \\
E(\Delta \ddot{Y})=-A E(\Delta \ddot{X})-2 A_{1} E(\Delta \dot{X})-A_{2} E(\Delta X)-B_{2} E(\Delta L)
\end{gathered}
$$

According to the theory that the random process and the derivative process are not related to each other, the output error matrix of the formula (2-2) to formula (2-7) can be obtained as follows:

$$
\begin{gathered}
V_{Y}=Z V_{X} Z^{T}+T V_{L} T^{T} \\
V_{\dot{Y}}=Z V_{\dot{X}} Z^{T}+Z_{1} V_{X} Z_{1}^{T}+T_{1} V_{L} T_{1}^{T} \\
V_{\ddot{Y}}=Z V_{\ddot{X}} Z^{T}+Z_{1} V_{\dot{X}} Z_{1}^{T}+Z_{2} V_{X} Z_{2}^{T}+T_{2} V_{L} T_{2}^{T}
\end{gathered}
$$

Where:

$$
\begin{aligned}
& V_{X}=\operatorname{diag}\left({\sigma_{x_{1}}}^{2},{\sigma_{x_{2}}}^{2}, \cdots, \sigma_{x_{m}}{ }^{2}\right) ; V_{\dot{X}}=\operatorname{diag}\left({\sigma_{\dot{x}_{1}}}^{2},{\sigma_{\dot{x}_{2}}}^{2}, \cdots{\sigma_{\dot{x}_{m}}}^{2}\right) \\
& V_{\ddot{X}}=\operatorname{diag}\left({\sigma_{\ddot{x}_{1}}}^{2},{\sigma_{\ddot{x}_{2}}}^{2}, \cdots,{\sigma_{\ddot{x}_{m}}}^{2}\right) ; V_{L}=\operatorname{diag}\left({\sigma_{l_{1}}}^{2},{\sigma_{l_{2}}}^{2}, \cdots,{\sigma_{l_{m}}}^{2}\right)
\end{aligned}
$$

The $V_{X}, V_{\dot{X}}, V_{\ddot{X}}$ vectors are determined by the displacement, velocity and acceleration of the input parameters, and the $V_{L}$ vector is determined by the effective structural parameters of the mechanism.

\section{Analysis of Comprehensive Motion Error of Six-bar Linkage Locking Mechanism}

In the locking mechanism, $\omega_{i}=\dot{\theta}_{i}$ and $\alpha_{i}=\ddot{\theta}_{i}$ respectively represent the angular velocity and angular acceleration of the mechanism component rod, the input angle $\theta_{2}$ is $90^{\circ}$, the input angular displacement error is 0 , and the variance is $6.5 \times 10^{-5} \mathrm{rad}^{2}$. The remaining lengths of the constituent rods are listed in Table 1 , with a large value of $r_{i}=1 \mathrm{~mm}(i=2, \cdots, 7)$ for the gap radius. The input error, rod length error, and mechanism output of the six-bar mechanism are all subject to a normal distribution [7], and the input speed of the mechanism linkage $l_{2}$ is $5 \mathrm{rad} / \mathrm{s}$. According to actual operating conditions and structural constraints, the agency operating angle is $45^{\circ} \sim 135^{\circ}$.

Table 1. Geometrical dimensions of various components in a six-bar locking mechanism $(\mathrm{mm})$

Linkage $l_{2} \quad$ Linkage $l_{3} \quad$ Linkage $l_{4} \quad$ Linkage $l_{5} \quad$ Linkage $l_{6} \quad$ Linkage $l_{12} \quad$ Linkage $l_{13}$

\begin{tabular}{lllllll}
$175 \pm 1$ & $330 \pm 2$ & $175 \pm 1$ & $1564 \pm 3$ & $175 \pm 1$ & $330 \pm 2$ & $1564 \pm 3$ \\
\hline
\end{tabular}


According to the above model, the motion error analysis results of the six-bar mechanism can be obtained by using MATLAB software to write the corresponding program of the mechanism motion error analysis. The output parameter of mechanism 6 is shown in Figure 4.

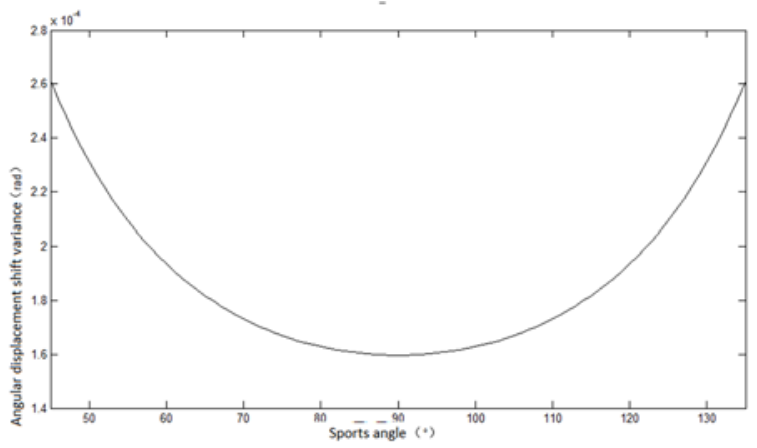

(a) Angular displacement variance curve

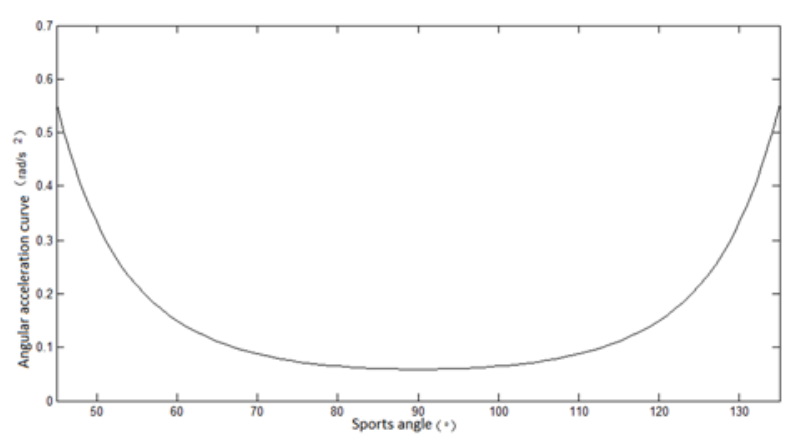

(c) Angular acceleration curve

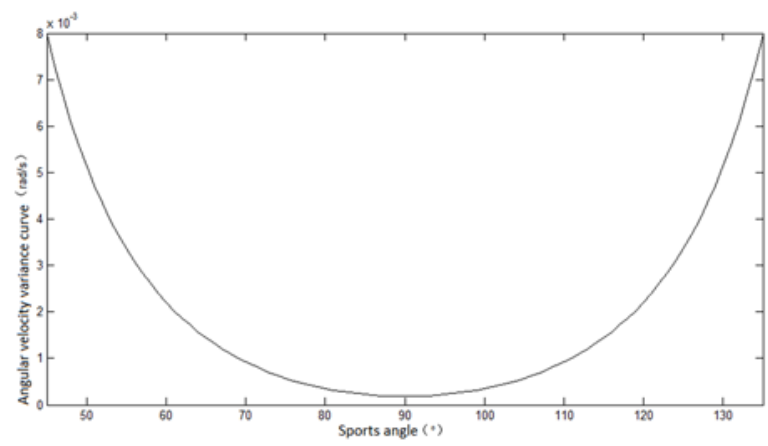

(b) Angular velocity variance curve

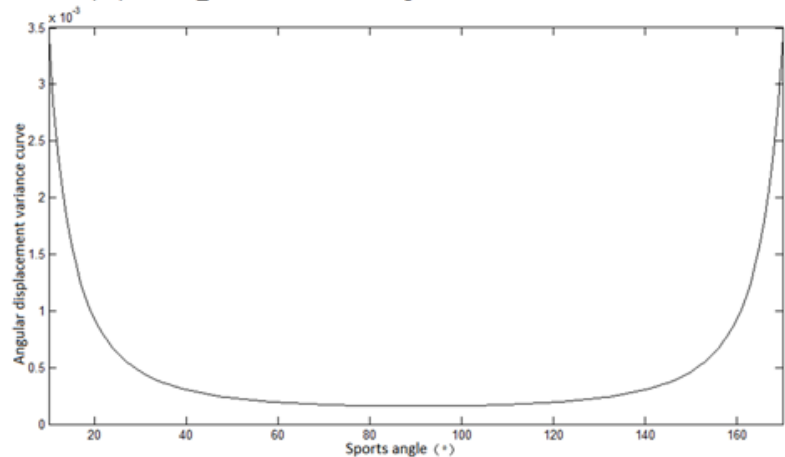

(d) Overall movement angular displacement variance

Figure 4. Output parameter variance curve of output member 6

From Fig. 4, it can be seen that the output parameters (angular displacement, angular velocity, angular acceleration) of the output member rod 6 change with the movement angle, the maximum angular displacement variance is $2.6123 e-4$, the maximum angular velocity variance value is $8 e-3$, the maximum angle The variance of acceleration is 0.6528 . From the change trend, the output variance of member 6 is the largest at $45^{\circ}$ and $135^{\circ}$, and the variance of the output parameter is the smallest at $90^{\circ}$.However, from the perspective of the motion variance of the overall movement angle, the movement angle is output between $45^{\circ}$ and $135^{\circ}$. The small parameter variance and the relatively gentle change can ensure that both the initial position and the working position have high motion accuracy. Therefore, the selection of such a motion angle region is reasonable.

\section{Conclusion}

In this paper, the composite hinge zero offset method is used to deal with the composite hinge problem. Based on this method, an effective rod length model with clearance is established. Finally, a six-link locking mechanism movement error analysis model is established by using the matrix method to comprehensively consider the factors of gap and rod length accuracy. The model was used to analyze the movement accuracy of the six-bar locking mechanism in the project. The analysis results show that the movement error model established in this paper can better analyze the movement error of the linkage mechanism with three-link composite hinge with clearance, and it is very suitable for computer programming operations.

\section{References}

[1]. Shi Z. Synthesis of mechanical error in spatial linkages based on reliability concept[J]. Mechanism \& Machine Theory, 1997, 32(32):255-259. 
[2]. Shi Z. Reliability-based analysis and synthesis of mechanical error for path generating linkages[J] Chinese Journal of Mechanical Engineering, 1997(2):130-135.

[3]. Wang Jun, Xiong Yuhua, Ruan Jinkui, et al. Probability Analysis of Motion Errors for Planar Linkage Mechanisms Considering Joint Backlash and Full-size Parameter Errors[J]. Mechanical Design, 2000, 17(8): 41-43.

[4]. Wang Chengzhi. Topological Representation and Automatic Kinematic Loop Analysis for Linkages with Multiple Joints[J]. Journal of agricultural machinery, 2009, 40(2): 199-203.

[5]. Zheng Wenwei, Wu Keyian. Mechanical Principles[M]. Southeast University Press, 2005.

[6]. Sun Zhili, Ji Guangzhen, Yan Yvtao, et al Mechanism Motion Reliability Design and Analysis Technology[M]. National Defense Industry Press, 2015.

[7]. Ye Qigen, Zhu Fengshi, Huang Jiaxian Mechanism accuracy [M]. Northwest Telecommunications Engineering Institute Press, 1986. 